\title{
Do irrelevant emotional stimuli impair or improve executive control?
}

\author{
Noga Cohen* and Avishai Henik \\ Department of Psychology, Zlotowski Center for Neuroscience, Ben-Gurion University of the Negev, Beer-Sheva, Israel \\ *Correspondence: nogac@post.bgu.ac.il
}

Our behavior is constantly influenced by emotional stimuli. These stimuli can enhance (i.e., improve) or impair performance, depending on their specific interaction with situational demands (Dolcos et al., 2011). This paper examines factors that mediate the influence of task-irrelevant negative stimuli on executive control (EC). We demonstrate how similar results of emotion-cognition interactions might be interpreted according to opposing theories, following the use of different analysis methods.

Executive control is responsible for monitoring, controlling, and regulating irrelevant information, in order to enable goal-directed behavior (Norman and Shallice, 1986). Recently, there is growing debate regarding the influence of negative stimuli on EC (Hu et al., 2012). Specifically, compared to neutral stimuli, negative stimuli were found to impair (i.e., elongated reaction times - RT), improve (i.e., facilitated RT), or have no influence (i.e., similar RT) on EC. Herein we will first suggest that descriptions of improved or impaired EC may be misleading, and then discuss three factors that modulate links between EC and emotion: available resources, attentional breadth, and top-down modulation.

\section{TACKLING THE TERMS IMPAIRED AND IMPROVED EC}

Two tasks widely used to examine EC are the Stroop (1935) and flanker (Eriksen and Eriksen, 1974). In the Stroop task, participants are presented with colored words written in colored ink and need to name the ink color and ignore the word. This task usually contains congruent, incongruent, and neutral targets (e.g., BLUE, RED, XXXX, respectively where the response should be "blue"). In the flanker task, participants need to respond to a target and ignore distracting stimuli. For example, in the arrow-flanker task, participants respond to the direction of the middle arrow and ignore the two flanker arrows. This task also contains congruent $(\rightarrow \rightarrow \rightarrow \rightarrow \rightarrow)$, incongruent $(\rightarrow \rightarrow \leftarrow \rightarrow \rightarrow)$, and neutral (-- $\rightarrow--)$ targets. Although the Stroop and flanker tasks differ in several aspects (Magen and Cohen, 2002), both create conflict situations during incongruent targets (although, see Goldfarb and Henik, 2007, for discussion of conflict also during congruent trials). This conflict situation activates EC mechanisms designed to detect and solve the conflict.

Studies presenting neutral and negative stimuli prior to Stroop or flanker tasks (Dennis and Chen, 2007a,b; Dennis et al., 2008; Hart et al., 2010; Cohen et al., 2011, 2012; O’Toole et al., 2011; Hu et al., 2012; Melcher et al., 2012) usually analyze the influence of these stimuli on the congruity effect (i.e., RT incongruent minus RT congruent). A larger congruity effect is usually interpreted as impaired EC, while a smaller congruity effect is usually interpreted as improved EC. However, this analysis does not distinguish between different effects of negative stimuli on congruent and incongruent targets separately. This distinction is important due to the presence of conflict (and hence, activation of EC) mainly during incongruent targets. Without showing a significant difference between negative and neutral stimuli influence on incongruent targets, it might be problematic to claim negative stimuli impair or improve EC. Importantly, this difference should not appear in congruent or neutral targets of the EC task (this would imply a main emotional effect on RT).

We recently showed that compared to neutral stimuli, negative stimuli delayed RTs for congruent flanker targets, but had no influence on incongruent targets (Cohen et al., 2011, 2012). Although these findings indicate a smaller congruity effect following negative stimuli, we did not interpret them as improved EC, but as attenuation of the emotional effect during conflict situations. Nevertheless, our paper was cited as evidence for improved EC (Birk et al., 2011). It is possible that this pattern (i.e., incongruent trials not affected by negative stimuli) is caused by improved EC. However, it is also possible that other mechanisms are responsible for this null effect (see Cohen et al., 2011; Hu et al., 2012, for debate regarding interpretation of their results). Similarly, interpretations of impaired EC may be misleading when data does not indicate delayed RTs for incongruent targets following negative compared to neutral stimuli.

Figure 1 illustrates typical results (not based on empirical data) that can be found in experiments presenting negative and neutral stimuli prior to an executive task. Figures 1A,B demonstrate results suggesting impaired (i.e., larger congruity effect) EC, while Figures 1C,D demonstrate results suggesting improved (i.e., smaller congruity effect) EC following presentation of negative stimuli. A similar congruity effect is found in Figures $\mathbf{1 A}, \mathbf{B}$, and a similar congruity effect is found in Figures 1C,D. However, these similar effects are produced in different ways (e.g., the impaired congruity effect in Figure 1A is due to elongated RT in negative incongruent trials, whereas the impaired congruity effect in Figure 1B is due to facilitated RT in negative congruent trials, with no change in the incongruent trials).

The following sections present three factors that might account for these different interaction patterns. In addition, we will demonstrate how similar results might be interpreted differently, depending on the analysis used.

\section{AVAILABLE RESOURCES}

According to the dual competition model (DCM), highly threatening stimuli impair EC since they consume resources required for resolving conflict (Pessoa, 2009). In accordance with this model, several studies demonstrated negative stimuli impair performance in a Stroop-like task (Hart et al., 

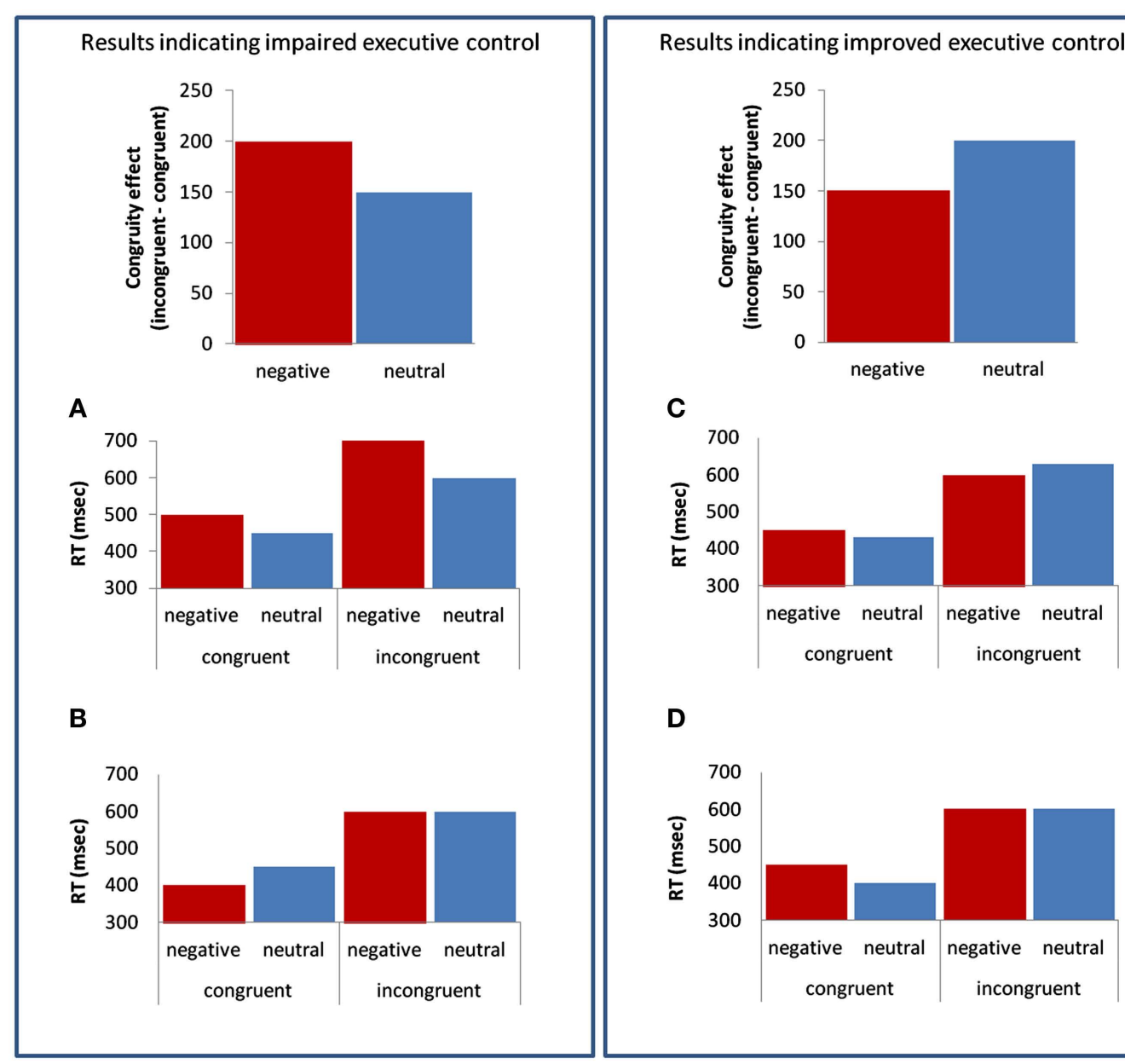

D

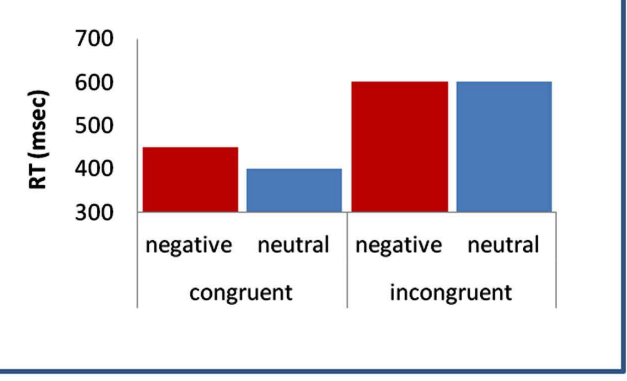

FIGURE 1 | Illustration of "impaired" (A,B) and "improved" (C,D) executive control following negative, compared to neutral, stimuli.

2010; Melcher et al., 2011; Padmala et al., 2011) and in the flanker task (Dennis et al., 2008). Although these studies reported impaired EC, close examination of the data of several of them may imply an alternative interpretation for the results.

Support for the DCM is found in studies reporting increased RTs for incongruent targets following negative compared to neutral stimuli (Hart et al., 2010; Melcher et al., 2011; see Figure 1A), thus demonstrating negative stimuli impair EC. In contrast, other studies interpreted results as being a consequence of impaired EC although their data did not show increased RTs for incongruent targets following negative stimuli. For example, examining RTs in the study of Dennis et al. (2008) may imply the increased congruity effect found after presentation of negative stimuli (and interpreted as impaired EC), resulted from facilitated RTs for negative compared to neutral stimuli in congruent trials, while RTs for incongruent trials following negative and neutral stimuli were very similar (Dennis et al., 2008; see Figure 1B). Findings of facilitation or lack of emotional interference in congruent trials (Dennis et al., 2008; Hart et al., 2010) are inconsistent with data showing delayed RTs in discrimination tasks following irrelevant emotional stimuli (Hartikainen et al., 2000; Buodo et al., 2002). Moreover, the DCM (Pessoa, 2009) would have predicted that resources devoted for processing emotional stimuli would cause delay both in congruent and in incongruent targets (although to a greater extent in incongruent targets; see Figure 1A, and Blair et al., 2007, for debate regarding this notion).

The study of Dennis et al. (2008) is an example of our claim that analyzing difference scores (i.e., congruity effect) might lead to incomplete or inaccurate interpretation of results. We suggest that under some circumstances emotional stimuli impair EC, thus supporting the DCM. However, there is also evidence emotional stimuli improve or have no influence on EC (see below). Hence, more data is needed to uncover the specific situations in which this impairment occurs.

\section{ATTENTIONAL BREADTH}

The DCM discussed above emphasizes the importance of available resources for solving a cognitive conflict. In contrast, attentional breadth theories (e.g., Easterbrook, 1959) emphasize the impact of negative information on attentional allocation. These theories claim negative stimuli narrow attention and hence, reduce interference of distracting or irrelevant information (Derryberry and Tucker, 1994; Chajut and Algom, 2003; van Steenbergen et al., 2011). 
Indeed, a smaller congruity effect following negative compared to neutral stimuli is often driven by facilitation of incongruent targets (see example in Figure 1C), as a result of reduced attention to distractors (in the flanker task) or the irrelevant dimension (in the Stroop task).

Evidence regarding improved EC following presentation of negative stimuli is found in Birk et al.'s (2011) study. The authors found reduced RTs for incongruent flanker targets following presentation of fearful compared to neutral faces (see Figure 1C). Several other studies also suggested emotion improves EC (Dennis and Chen, 2007b; Hu et al., 2012; Melcher et al., 2012). However, in contrast to Birk et al.'s study, the reduced congruity effect in the latter studies did not result from reduced RTs in incongruent targets. For example, in a Stroop-like task, Hu et al. (2012) found that compared to neutral situations, negative situations (shock anticipation) delayed RTs in congruent trials, but no difference between neutral and negative situations was found in incongruent trials (see Figure 1D). The authors suggested the overall slowdown following shock anticipation was contrasted by the facilitation effect caused by attentional narrowing. Importantly, they based their claim on results showing delayed RTs during neutral Stroop trials (e.g., XXX where the response should be "red"). As discussed in their paper, adding neutral Stroop trials may help unravel different effects of attentional narrowing and available resources on EC.

Hence, it seems that under specific circumstances, negative stimuli improve EC. In addition, it seems that findings of a null emotional effect in incongruent targets are sometimes interpreted as improved EC and sometimes as impaired EC (as described earlier). We suggest this null emotional effect in incongruent targets may result from a top-down regulation mechanism (see supporting explanation below).

\section{TOP-DOWN MODULATION}

Decreased emotional response during or following executive activation led to the suggestion EC can trigger top-down mechanisms that inhibit the effect of emotion (Etkin et al., 2006; Blair et al., 2007; Cohen et al., 2011, 2012). Etkin et al. (2006) presented a modified Stroop task where participants had to respond to the emotionality of a face (happy or fearful) and ignore a superimposed word (that was congruent or incongruent with the face emotion). They demonstrated a conflict adaptation effect in which the response for an incongruent target attenuated the response of the following incongruent target. This decrease in emotional conflict was related to a connection between brain areas known to be activated during conflict monitoring (e.g., anterior cingulate cortex) and emotional areas (e.g., amygdala). Similarly, we recently showed that when an executive task precedes an emotional stimulus, incongruent trials can attenuate emotional response both behaviorally (Cohen et al., 2011, 2012; see Figure 1D) and physiologically (Cohen and Henik, Submitted). In addition, there is evidence that this inhibitory connection characterizes healthy individuals and is deficient in people suffering from depression (Johnstone et al., 2007; for review, see Rogers et al., 2004) or anxiety (Bishop et al., 2004; Etkin et al., 2010). Taken together, there is strong evidence that activation of EC might result in decreased emotional response and hence, in a diminished emotional effect during incongruent targets.

\section{CONCLUSION}

We presented several factors that might account for the various relationships between emotion and EC. In addition, we suggested that the use of difference scores (i.e., the congruity effect) might lead to different interpretations, even in face of similar data sets. We offer two important conclusions:

1. The effect of emotion on EC is modulated by several factors. Negative stimuli impair EC if they consume resources available for resolving the conflict, improve EC if attention is narrowed, or have no influence on EC if top-down processes are activated. Additional factors, such as relevance of the negative stimuli to current goals (Kanske and Kotz, 2011a,b; see also Kanske, in press, in this Research Topic), task demands (Shafer et al., 2012), and individual differences, may also modulate the effects of emotion on EC (for review, see Okon-Singer et al., 2012). More research is required to unravel how all these factors are orchestrated to shape the emotionexecutive relationship.
2. Researchers tend to analyze the influence of emotion on the congruity effect and by doing so, miss important information. A good example for inconsistent interpretation occurs when researchers find a null emotional effect for incongruent targets. This null effect is sometimes interpreted as impaired, and sometimes as improved EC, depending on the effect of emotion on congruent trials (compare Figures 1B,D, respectively). Hu et al. (2012) suggested that adding neutral trials to the EC task might help distinguish between impairing and enhancing effects. However, as discussed above this null effect may also result from top-down regulation of the emotional system. This top-down effect should be directly examined by conducting a sequential analysis (Cohen et al., 2011) or by presenting the EC task before the emotional stimuli (Cohen et al., 2012).

Future studies should strive to uncover the specific factors that modulate emotional effect on conflict vs. non-conflict situations. Defining the interactions between these factors could uncover how emotion and EC are mutually linked in order to generate adaptive behavior. On a broader perspective, the use of difference scores, such as the congruity effect, in various cognitive domains (e.g., spatial attention, implicit attitudes, affective priming) may lead to an incomplete view of emotion-cognition interactions, thus hindering the research in this field.

\section{REFERENCES}

Birk, J. L., Dennis, T.A., Shin, L. M., and Urry, H. L. (2011). Threat facilitates subsequent executive control during anxious mood. Emotion 11, 1291-1304.

Bishop, S., Duncan, J., Brett, M., and Lawrence, A. D. (2004). Prefrontal cortical function and anxiety: controlling attention to threat-related stimuli. Nat. Neurosci. 7, 184-188.

Blair, K. S., Smith, B. W., Mitchell, D. G. V., Morton, J., Vythilingam, M., Pessoa, L., Fridberg, D., Zametkin, A., Nelson, E. E., Drevets, W. C., Pine, D. S., Martin, A., and Blair, R. J. R. (2007). Modulation of emotion by cognition and cognition by emotion. Neuroimage 35, 430-440.

Buodo, G., Sarlo, M., and Palomba, D. (2002).Attentional resources measured by reaction times highlight differences within pleasant and unpleasant, high arousing stimuli. Motiv. Emot. 26, 123-138.

Chajut, E., and Algom, D. (2003). Selective attention improves under stress: implications for theories of social cognition. J. Pers. Soc. Psychol. 85, 231-248. 
Cohen, N., Henik, A., and Mor, N. (2011). Can emotion modulate attention? Evidence for reciprocal links in the attentional network test. Exp. Psychol. 58, 171-179.

Cohen, N., Henik, A., and Moyal, N. (2012). Executive control attenuates emotional effects - for high reappraisers only? Emotion (in press).

Dennis, T. A., Chen, C., and McCandliss, B. D. (2008). Threat related attentional biases: an analysis of three attention systems. Depress. Anxiety 25, E1-E10.

Dennis, T. A., and Chen, C.-C. (2007a). Emotional face processing and attention performance in three domains: neurophysiological mechanisms and moderating effects of trait anxiety. Int. J. Psychophysiol. 65, 10-19.

Dennis, T.A., and Chen, C.-C.(2007b). Neurophysiological mechanisms in the emotional modulation of attention: the interplay between threat sensitivity and attentional control. Biol. Psychol. 76, 1-10.

Derryberry, D., and Tucker, D. M. (1994). "Motivating the focus of attention," in The Heart's Eye: Emotional Influences in Perception and Attention, eds P. M. Niedenthal and S. Kitayama (San Diego: Academic Press), 167-196.

Dolcos, F., Iordan, A. D., and Dolcos, S. (2011). Neural correlates of emotion-cognition interactions: a review of evidence from brain imaging investigations. J. Cogn. Psychol. (Hove) 23, 669-694.

Easterbrook, J. A. (1959). The effect of emotion on cue utilization and the organization of behavior. Psychol. Rev. 66, 183-201.

Eriksen, B. A., and Eriksen, C. W. (1974). Effects of noise letters upon the identification of a target letter in a nonsearch task. Percept. Psychophys. 16, 143-149.

Etkin, A., Egner, T., Peraza, D. M., Kandel, E. R., and Hirsch, J. (2006). Resolving emotional conflict: a role for the rostral anterior cingulate cortex in modulating activity in the amygdala. Neuron 51, 871-882.

Etkin, A., Prater, K. E., Hoeft, F., Menon, V., and Schatzberg, A. F. (2010). Failure of anterior cingulate activation and connectivity with the amygdala during implicit regulation of emotional processing in generalized anxiety disorder. Am. J. Psychiatry 167, 545-554.
Goldfarb, L., and Henik, A. (2007). Evidence for task conflict in the Stroop effect. J. Exp. Psychol. Hum. Percept. Perform. 33, 1170-1176.

Hart, S. J., Green, S. R., Casp, M., and Belger, A. (2010). Emotional priming effects during Stroop task performance. Neuroimage 49, 2662-2670.

Hartikainen, K. M., Ogawa, K. H., and Knight, R. T. (2000). Transient interference of right hemispheric function due to automatic emotional processing. Neuropsychologia 38, 1576-1580.

Hu, K., Bauer, A., Padmala, S., and Pessoa, L. (2012). Threat of bodily harm has opposing effects on cognition. Emotion 12, 28-32.

Johnstone, T., Van Reekum, C. M., Urry, H. L., Kalin, N. H., and Davidson, R. J. (2007). Failure to regulate: counterproductive recruitment of top-down prefrontal-subcortical circuitry in major depression. J. Neurosci. 27, 8877-8884.

Kanske, P. (2012). On the influence of emotion on conflict processing. Front. Integr. Neurosci. (in press).

Kanske, P., and Kotz, S. A. (2011a). Emotion speeds up conflict resolution: a new role for the ventral anterior cingulate cortex? Cereb. Cortex 21, 911-919.

Kanske, P., and Kotz, S. A. (2011b). Emotion triggers executive attention: anterior cingulate cortex and amygdala responses to emotional words in a conflict task. Hum. Brain Mapp. 32, 198-208.

Magen, H., and Cohen, A. (2002). Action-based and vision-based selection of input: two sources of control. Psychol. Res. 66, 247-259.

Melcher, T., Born, C., and Gruber, O. (2011). How negative affect influences neural control processes underlying the resolution of cognitive interference: an event-related fMRI study. Neurosci. Res. 70, 415-427.

Melcher, T., Obst, K., Mann, A., Paulus, C., and Gruber, O. (2012). Antagonistic modulatory influences of negative affect on cognitive control: reduced and enhanced interference resolution capability after the induction of fear and sadness. Acta Psychol. (Amst.) 139, 507-514.

Norman, D., and Shallice, T. (1986). "Attention to action: willed and automatic control of behavior," in Consciousness and Self-Regulation, Vol. 4, eds R. J.
Davidson, G. E. Schwartz, and D. Shapiro (New York: Plenum Press) 1-18.

Okon-Singer, H., Lichtenstein-Vidne, L., and Cohen, N. (2012). Dynamic modulation of emotional processing. Biol. Psychol. doi:10.1016/j.biopsycho.2012.05.010

O'Toole, L. J., DeCicco, J. M., Hong, M., and Dennis, T. A. (2011). The impact of task-irrelevant emotional stimuli on attention in three domains. Emotion 11, 1322-1330.

Padmala, S., Bauer, A., and Pessoa, L. (2011). Negative emotion impairs conflict-driven executive control. Front. Psychol. 2:192. doi: 10.3389/fpsyg.2011.00192

Pessoa, L. (2009). How do emotion and motivation direct executive control? Trends Cogn. Sci. (Regul. Ed.) 13, 160-166.

Rogers, M. A., Kasai, K., Koji, M., Fukuda, R., Iwanami, A., Nakagome, K., Fukuda, M., and Kato, N. (2004). Executive and prefrontal dysfunction in unipolar depression: a review of neuropsychological and imaging evidence. Neurosci. Res. 50, 1-11.

Shafer, A. T., Matveychuk, D., Penney, T., O’Hare, A. J., Stokes, J., and Dolcos, F. (2012). Processing of emotional distraction is both automatic and modulated by attention: evidence from an event-related fMRI investigation. J. Cogn. Neurosci. 24, 1233-1252.

Stroop, J. R. (1935). Studies of interference in serial verbal reactions. J. Exp. Psychol. 18, 643-662.

van Steenbergen, H., Band, G. P. H., and Hommel, B. (2011). Threat but not arousal narrows attention: evidence from pupil dilation and saccade control. Front. Psychol. 2:281. doi: 10.3389/ fpsyg.2011.00281

Received:20 March 2012; accepted: 28 May 2012; published online: 18 June 2012.

Citation: Cohen N and HenikA (2012) Do irrelevant emotional stimuli impair or improve executive control? Front. Integr. Neurosci. 6:33. doi: 10.3389/fnint.2012.00033

Copyright (C) 2012 Cohen and Henik. This is an openaccess article distributed under the terms of the Creative Commons Attribution Non Commercial License, which permits non-commercial use, distribution, and reproduction in other forums, provided the original authors and source are credited. 